\title{
Gravitational Fields of Conical Mass Distributions
}

\author{
Chifu Ebenezer Ndikilar \\ Physics Department, Faculty of Science, Federal University Dutse, P.M.B. 7156, Dutse, Jigawa State 720222, Nigeria \\ Correspondence should be addressed to Chifu Ebenezer Ndikilar; ebenechifu@yahoo.com
}

Received 20 February 2013; Revised 11 March 2013; Accepted 19 March 2013

Academic Editor: Maddalena Mantovani

Copyright (C) 2013 Chifu Ebenezer Ndikilar. This is an open access article distributed under the Creative Commons Attribution License, which permits unrestricted use, distribution, and reproduction in any medium, provided the original work is properly cited.

\begin{abstract}
The gravitational field of conical mass distributions is formulated using the general theory of relativity. The gravitational metric tensor is constructed and applied to the motion of test particles and photons in this gravitational field. The expression for gravitational time dilation is found to have the same form as that in spherical, oblate spheroidal, and prolate spheroidal gravitational fields and hence confirms an earlier assertion that this gravitational phenomena is invariant in form with various mass distributions. It is shown using the pure radial equation of motion that as a test particle moves closer to the conical mass distribution along the radial direction, its radial speed decreases.
\end{abstract}

\section{Introduction}

In recent articles [1-4], we introduced an approach of studying gravitational fields of various mass distributions as extensions of Schwarzschild's method. Of interest in this article is the gravitational field of conical mass distributions placed in empty space. Sputnik III, the third Soviet satellite launched on May 15, 1958 has a conical shape. This study is aimed at studying the behaviour of test particles and photons in the vicinity of conically shaped objects placed in empty space such as Sputnik III.

\section{Gravitational Metric Tensors}

It is well known $[5,6]$ that the general relativistic metric tensor for flat space-time (empty space without mass) is invariant (invariance of the line element) and can be obtained in any orthogonal curvilinear coordinate $(t, u, v, w)$ by the transformation $(t, x, y, z) \rightarrow(t, u, v, w)$. It is worth noting that the metric tensor obtained through this standard procedure

(i) satisfies Einstein's field equations a priori and

(ii) yields the expected equations of motion for test particles and photons in flat space-time.

Using these crucial facts, we now realize that choosing the particular orthogonal curvilinear coordinate $(u, v, w)$ corresponding to the geometry of the body facilitates the formulation of boundary conditions on the universal gravitational scalar potential which is always expected to be a part of the metric tensor. Thus, transforming Schwarzschild's metric into the particular orthogonal curvilinear coordinate using the invariance of the line element subject to the fact that the arbitrary function in the metric tensor transforms as $f(t, r, \theta, \phi) \rightarrow f(t, u, v, w)$ yields the metric tensor for the mass distribution of the orthogonal curvilinear coordinate. Thus, the arbitrary function $f$ is determined by the mass or pressure distribution and hence possess symmetries imposed by the latter a priori. In approximate gravitational fields, the arbitrary function is equal to the gravitational scalar potential.

Thus, to obtain the metric tensor for a homogeneous conical mass distribution placed in empty space in conical coordinates:

(1) coordinates are transformed from spherical polar $(r, \theta, \phi)$ to conical $(r, v, w)$ on the right hand side of Schwarzschild's world line element;

(2) the arbitrary function $f(r)$ in Schwarzschild's field is transformed to $f^{\prime}(r)$ in the field of a homogeneous conical mass. 
Now, the spherical polar coordinates $(r, \theta, \phi)$ are related to the Cartesian coordinates $(x, y, z)$ as follows:

$$
\begin{gathered}
r=\left(x^{2}+y^{2}+z^{2}\right)^{1 / 2} \\
\theta=\cos ^{-1}\left(\frac{z}{\left(x^{2}+y^{2}+z^{2}\right)^{1 / 2}}\right), \\
\phi=\tan ^{-1}\left(\frac{y}{x}\right) .
\end{gathered}
$$

Also the Cartesian coordinates are related to the conical coordinates $(r, v, w)$ as follows:

$$
\begin{gathered}
x=\frac{1}{\alpha} r\left(\alpha^{2}-v^{2}\right)^{1 / 2}\left(\alpha^{2}+w^{2}\right)^{1 / 2}, \\
y=\frac{1}{\beta} r\left(\beta^{2}+v^{2}\right)^{1 / 2}\left(\beta^{2}-w^{2}\right)^{1 / 2}, \\
z=\frac{1}{\alpha \beta} r v w,
\end{gathered}
$$

where $\alpha^{2}+\beta^{2}=1$.

Expressing the spherical coordinates in terms of the conical coordinates, we obtain

$$
\begin{gathered}
\theta(r, v, w)=\cos ^{-1}\left(\frac{v w}{\alpha \beta}\right), \\
\phi(r, v, w)=\tan ^{-1}\left[\frac{\beta\left(\alpha^{2}-v^{2}\right)^{1 / 2}\left(\alpha^{2}+w^{2}\right)^{1 / 2}}{\alpha\left(\beta^{2}+v^{2}\right)^{1 / 2}\left(\beta^{2}-w^{2}\right)^{1 / 2}}\right] .
\end{gathered}
$$

Now, consider a spherically symmetric mass distribution with invariant world line element (Schwarzschild's line element) in the exterior region given [1] as

$$
\begin{aligned}
c^{2} d \tau^{2}= & c^{2}\left(1+\frac{2 f(r)}{c^{2}}\right) d t^{2}-\left(1+\frac{2 f(r)}{c^{2}}\right)^{-1} d r^{2} \\
& -r^{2} d \theta^{2}-r^{2} \sin ^{2} \theta d \phi^{2},
\end{aligned}
$$

where $f(r)$ generally is an arbitrary function determined by the distribution of mass or pressure and possess all the symmetries of the distribution. In approximate gravitational fields, $f(r)$ is equivalent to the gravitational scalar potential function exterior to a massive symmetric sphere.

Let the spherically symmetric mass distribution be transformed by deformation into a conical mass distribution in such a way that it retains its density and total mass. Thus, the general relativistic invariant world line element exterior to a spherical symmetric body is tensorially equivalent to that of a conical mass distribution and related by the transformation from spherical to conical coordinates. Therefore, to obtain the invariant line element exterior to conical mass distributions, we transform coordinates on the right hand side of (5), from spherical to conical coordinates and $f(r) \rightarrow f^{\prime}(r)$, the arbitrary function determined by the conical mass.

From (3), we have

$$
d \theta=\frac{\partial \theta}{\partial r} d r+\frac{\partial \theta}{\partial v} d v+\frac{\partial \theta}{\partial w} d w
$$

where

$$
\begin{gathered}
\frac{\partial \theta}{\partial v}=-\frac{w}{\left(\alpha^{2} \beta^{2}-v^{2} w^{2}\right)^{1 / 2}} \\
\frac{\partial \theta}{\partial w}=-\frac{v}{\left(\alpha^{2} \beta^{2}-v^{2} w^{2}\right)^{1 / 2}}, \\
\frac{\partial \theta}{\partial r}=0 .
\end{gathered}
$$

Also from (4), we have

$$
d \phi=\frac{\partial \phi}{\partial r} d r+\frac{\partial \phi}{\partial v} d v+\frac{\partial \phi}{\partial w} d w
$$

where

$$
\begin{gathered}
\frac{\partial \phi}{\partial v}=-\frac{v^{3} w^{2}\left(\alpha^{2}+w^{2}\right)^{1 / 2}\left(\alpha^{2}-v^{2}\right)^{-1 / 2}}{\alpha^{3} \beta\left(\beta^{2}+v^{2}\right)^{3 / 2}\left(\beta^{2}-w^{2}\right)^{1 / 2}}, \\
\frac{\partial \phi}{\partial w}=\frac{v^{2} w^{3}\left(\alpha^{2}-v^{2}\right)^{1 / 2}\left(\alpha^{2}+w^{2}\right)^{-1 / 2}}{\alpha^{3} \beta\left(\beta^{2}-w^{2}\right)^{3 / 2}\left(\beta^{2}+v^{2}\right)^{1 / 2}}, \\
\frac{\partial \phi}{\partial r}=0 .
\end{gathered}
$$

Substituting (3), (6), and (8) into (5), the nonzero components of the covariant metric tensor exterior to conical mass distributions are obtained as

$$
\begin{gathered}
g_{00}=\left(1+\frac{2}{c^{2}} f^{\prime}(r)\right), \\
g_{11}=-\left(1+\frac{2}{c^{2}} f^{\prime}(r)\right)^{-1}, \\
g_{22}=-\frac{(\alpha \beta r w)^{2}}{\left(\alpha^{2} \beta^{2}-v^{2} w^{2}\right)^{2}} \\
-\frac{r^{2} v^{2}\left(\alpha^{2}+w^{2}\right)\left(\beta^{2}-w^{2}\right)}{\left(\alpha^{2} \beta^{2}-v^{2} w^{2}\right)\left(\alpha^{2}+v^{2}\right)\left(\beta^{2}-v^{2}\right)}, \\
g_{23} g_{32}=-\frac{(\alpha \beta r)^{2} v w}{\left(\alpha^{2} \beta^{2}-v^{2} w^{2}\right)^{2}} \\
g_{33}=-\frac{(\alpha \beta r w)^{2}}{\left(\alpha^{2} \beta^{2}-v^{2} w^{2}\right)^{2}} \\
-\frac{r^{2} w^{2}\left(\alpha^{2}-v^{2}\right)\left(\beta^{2}-v^{2}\right)}{\left(\alpha^{2} \beta^{2}-v^{2} w^{2}\right)\left(\alpha^{2}+w^{2}\right)\left(\beta^{2}-w^{2}\right)} .
\end{gathered}
$$


The nonzero components of the contravariant metric tensor for this gravitational field are obtained using tensor analysis as

$$
\begin{gathered}
g^{00}=\frac{1}{g_{00}}, \\
g^{11}=\frac{1}{g_{11}} \\
g^{22}=\frac{g_{33}}{g_{22} g_{33}-\left(g_{23}\right)^{2}}, \\
g^{23} \equiv g^{32}=\frac{g_{23}}{g_{22} g_{33}-\left(g_{23}\right)^{2}}, \\
g^{33}=\frac{g_{22}}{g_{22} g_{33}-\left(g_{23}\right)^{2}} .
\end{gathered}
$$

Thus, the metric tensor in this gravitational field unlike Schwarzschild's metric has six nonzero components. The contravariant metric components $g^{00}$ and $g^{11}$ are simply the reciprocal of their covariant counterparts. The remaining nonzero components of the contravariant metric have a common denominator.

\section{Motion of Test Particles}

The affine connection coefficients of the gravitational field are obtained using tensor analysis, and the motion of test particles in the vicinity of a conical mass distribution is studied using

$$
\frac{d^{2} x^{\mu}}{d \tau^{2}}+\Gamma_{\nu \lambda}^{\mu}\left(\frac{\partial x^{\nu}}{\partial \tau}\right)\left(\frac{\partial x^{\lambda}}{\partial \tau}\right)=0
$$

Setting $\mu=0$ in (12), we obtain the time equation of motion as

$$
\ddot{t}+\frac{2}{c^{2}}\left[1+\frac{2}{c^{2}} f^{\prime}(r)\right]^{-1} \frac{d f^{\prime}(r)}{d r} \dot{t} \dot{r}=0,
$$

where the dot represents differentiation with respect to proper time $\tau$. Solving (13) yields the expression for gravitational time dilation in this gravitational field as

$$
\dot{t}=\left[1+\frac{2}{c^{2}} f^{\prime}(r)\right]^{-1}
$$

It has the same form as that obtained in the vicinity of homogeneous spherical [1], prolate spheroidal [3], and oblate spheroidal $[2,4]$ mass distributions.Thus, only motion along the radial direction affects gravitational time dilation.
Similarly, setting $\mu=1$ in (12) yields the radial equation of motion as

$$
\begin{aligned}
\ddot{r}-[1 & \left.+\frac{2}{c^{2}} f^{\prime}(r)\right] \frac{d f^{\prime}(r)}{d r} \dot{t}^{2} \\
+ & \frac{2}{c^{2}}\left[1+\frac{2}{c^{2}} f^{\prime}(r)\right]^{-1} \frac{d f^{\prime}(r)}{d r} \dot{r}^{2}-\dot{v}^{2}\left[1+\frac{2}{c^{2}} f^{\prime}(r)\right] \\
& \times\left\{\frac{(\alpha \beta w)^{2} r}{(\alpha \beta)^{2}-(v w)^{2}}\right. \\
& \left.+\frac{v^{2} r\left(\alpha^{2}+w^{2}\right)\left(\beta^{2}-w^{2}\right)}{\left(\alpha^{2}+v^{2}\right)\left(\beta^{2}-v^{2}\right)\left[\left(\alpha^{2}+w^{2}\right)\left(\beta^{2}-w^{2}\right)\right]}\right\}=0 .
\end{aligned}
$$

To obtain a first approximation to the solution of (15), pure radial motion can be studied. For pure radial motion $\dot{t}=0$, $\dot{v}=0$, and (15) reduces to

$$
\ddot{r}+\frac{2}{c^{2}}\left[1+\frac{2}{c^{2}} f^{\prime}(r)\right]^{-1} \frac{d f^{\prime}(r)}{d r} \dot{r}^{2}=0 .
$$

Solving the pure radial equation yields solution as

$$
\dot{r}=A\left[1+\frac{2}{c^{2}} f^{\prime}(r)\right]^{-1},
$$

where $A$ is a constant of motion. This shows that $\dot{r}$ varies inversely with $f^{\prime}(r)$. Thus, an increase in the gravitational potential retards motion in the pure radial direction, and hence as a test particle moves closer to the conical mass distribution along the radial direction, its radial speed decreases. An astronomical expedition can be undertaken in the near future at the vicinity of Sputnik III to confirm this interesting results.

Also, setting $\mu=2$ and $\mu=3$, the respective $v$ and $w$ equations of motion are obtained as

$$
\begin{gathered}
\ddot{v}+2 \Gamma_{12}^{2} \dot{r} \dot{v}+2 \Gamma_{13}^{2} \dot{r} \dot{w}+\Gamma_{22}^{2} \dot{v}^{2}+2 \Gamma_{23}^{2} \dot{v} \dot{w}+\Gamma_{33}^{2} \dot{w}^{2}=0, \\
\ddot{w}+2 \Gamma_{12}^{3} \dot{r} \dot{v}+2 \Gamma_{13}^{3} \dot{r} \dot{w}+2 \Gamma_{23}^{3} \dot{v} \dot{w}+\Gamma_{33}^{3} \dot{w}^{2}=0 .
\end{gathered}
$$

\section{Orbits in the Vicinity of a Conical Mass Distribution}

The Langrangian for any gravitational field is defined [7] as

$$
L \equiv \frac{1}{c}\left(-g_{\alpha \beta} \frac{d x^{\alpha}}{d \tau} \frac{d x^{\beta}}{d \tau}\right)^{1 / 2},
$$

which can be written explicitly in this gravitational field as

$$
\begin{gathered}
L=\frac{1}{c}\left[-g_{00}\left(\frac{d x^{0}}{d \tau}\right)^{2}-g_{11}\left(\frac{d x^{1}}{d \tau}\right)^{2}-g_{22}\left(\frac{d x^{2}}{d \tau}\right)^{2}\right. \\
\left.-g_{33}\left(\frac{d x^{3}}{d \tau}\right)^{2}-2 g_{23}\left(\frac{d x^{2}}{d \tau}\right)\left(\frac{d x^{3}}{d \tau}\right)\right] .
\end{gathered}
$$


Now, consider spatial motion along the radial direction only, then the Langrangian reduces to

$$
L=\frac{1}{c}\left[-g_{00}\left(\frac{d x^{0}}{d \tau}\right)^{2}-g_{11}\left(\frac{d x^{1}}{d \tau}\right)^{2}\right]
$$

or more explicitly as

$$
L=-c\left(1+\frac{2}{c^{2}} f^{\prime}(r)\right) \dot{t}^{2}+\frac{1}{c}\left(1+\frac{2}{c^{2}} f^{\prime}(r)\right)^{-1} \dot{r}^{2} .
$$

Transforming the radial coordinate $r$ to the angular coordinate $\omega$ using $r=r(\omega)$ and defining $u(\omega)=1 / r(\omega)$, we have

$$
\dot{r}=-\frac{1}{1+u^{2}} \frac{d u}{d \omega} .
$$

Substituting (23) into (22) and using the fact that $L=1$ for time-like orbits and $L=0$ for null orbits, we obtain

$$
\begin{gathered}
\frac{d^{2} u}{d \omega^{2}}-\frac{2 u}{1+u^{2}} \frac{d u}{d \omega}=\frac{\left(1+u^{2}\right)^{2}}{c} \frac{d f(\omega)}{d u} \\
\frac{d^{2} u}{d \omega^{2}}-\frac{2 u}{1+u^{2}} \frac{d u}{d \omega}=0
\end{gathered}
$$

as the respective planetary equation of motion and photon equation of motion in the vicinity of a conical mass distribution placed in empty space. Equation (24) can be used to study the motion of orbiting particles moving in the vicinity of conical masses.

\section{Remarks and Conclusion}

The results obtained in this paper have paved the way for the study of gravitational fields of conical mass distributions like Sputnik III. The immediate consequences of the results obtained in this paper are as follows.

(1) Einstein's geometrical field equations can be constructed using our metric tensor. The striking fact about these field equations is that they have only one unknown $f^{\prime}(r)$ which is determined by the mass or pressure distribution. Thus, the field equations can be easily solved and explicit expressions obtained for $f^{\prime}(r)$.

(2) The planetary equation of motion and the photon equation of motion can be solved to describe the motion of test particles and photons in the vicinity of conical masses. These equations are opened up for further research work and astrophysical interpretation.

(3) In approximate gravitational fields, the arbitrary function $f^{\prime}(r)$ can be conveniently equated to the gravitational scalar potential exterior to a conical mass. Thus, if the complete expression of $f^{\prime}(r)$ is obtained from the field equations, it will depict a hither to unknown generalization of the gravitational scalar potential function for this field.

(4) Other gravitational phenomena such as gravitational spectral shift could be studied in the vicinity of conical masses.

\section{References}

[1] E. N. Chifu and S. X. K. Howusu, "Gravitational radiation and propagation field equation exterior to astrophysically real or hypothetical time varying distributions of mass within regions of spherical geometry," Physics Essays, vol. 22, no. 1, pp. 73-77, 2009.

[2] E. N. Chifu, A. Usman, and O. C. Meludu, "Orbits in homogeneous oblate spheroidal gravitational space time," Progress in Physics, vol. 3, pp. 49-53, 2009.

[3] E. N. Chifu, A. Usman, and O. C. Meludu, "Exact analytical solutions of Einstein's gravitational field equations in static homogeneous prolate spheroidal space-time," African Journal of Mathematical Physics, vol. 9, pp. 25-42, 2010.

[4] E. N. Chifu, "Gravitational fields exterior to homogeneous spheroidal masses," Abraham Zelmanov Journal of General Relativity, Gravitation and Cosmology, vol. 5, pp. 31-67, 2012.

[5] P. G. Bergmann, Introduction to the Theory of Relativity, Prentice Hall, New Delhi, India, 1987.

[6] S. Weinberg, Gravitation and Cosmology, John Wiley \& Sons, New York, NY, USA, 1972.

[7] K. S. D. Peter, "An introduction to tensors and relativity," Cape Town, pp. 51-110, 2000. 

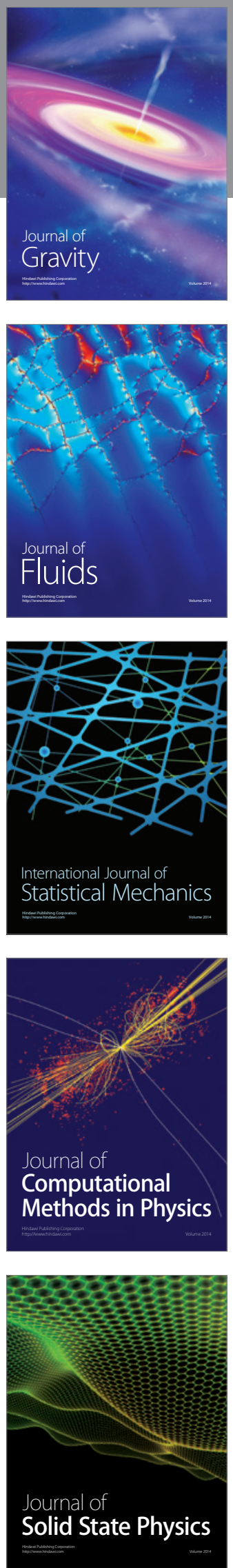

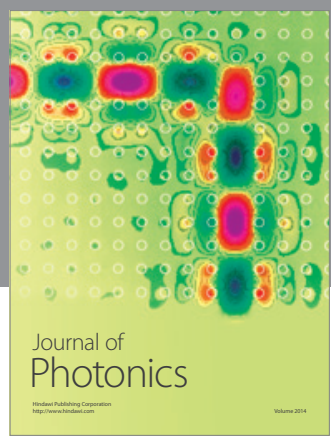

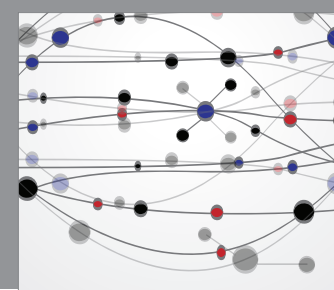

The Scientific World Journal

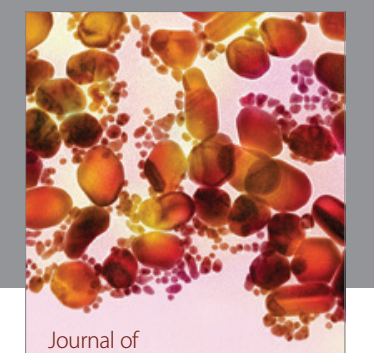

Soft Matter
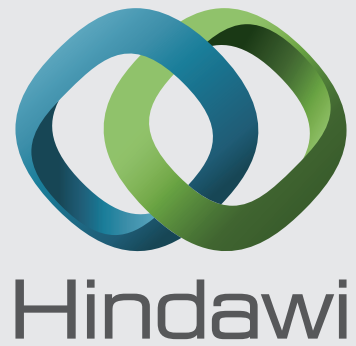

Submit your manuscripts at

http://www.hindawi.com
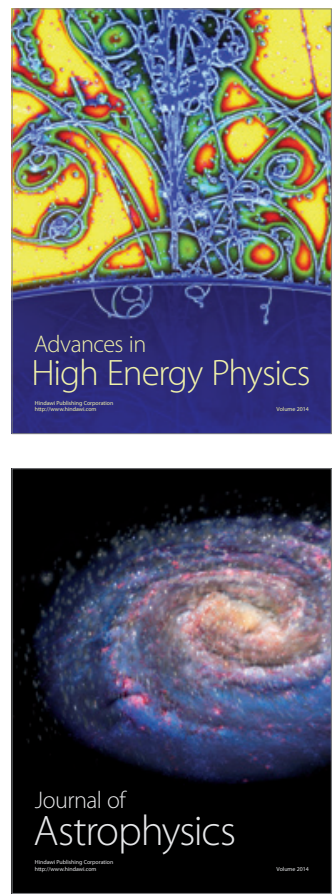
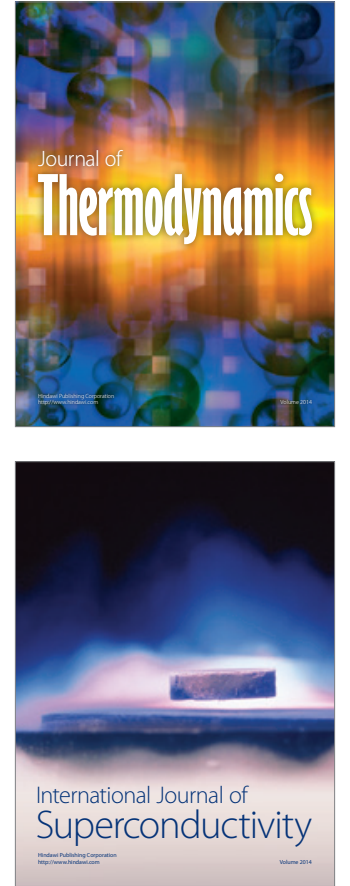
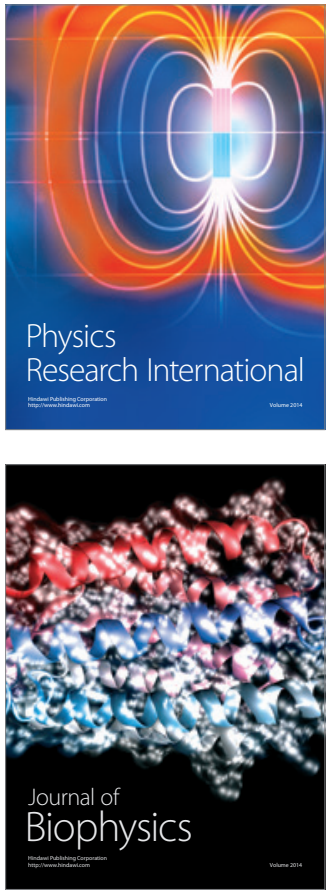
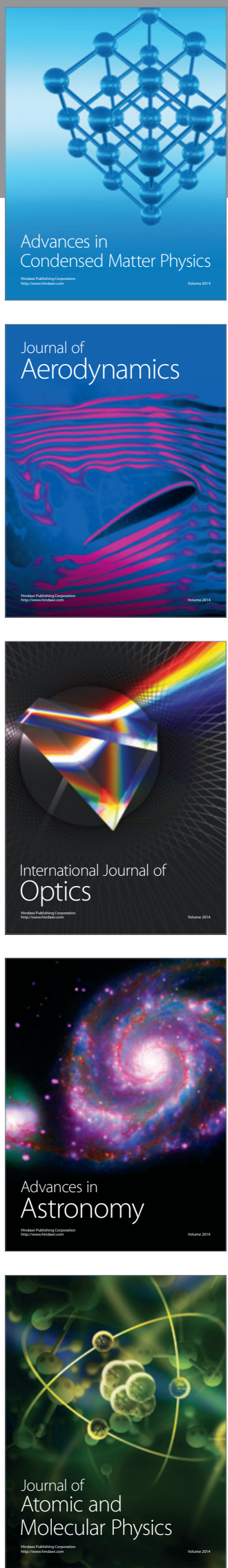